mit einer starken Quecksilberquarzlampe, so tritt sofort Entfärbung und Chlorwasserstoffentwicklung ein. Während des Einleitens bleibt die Flüssigkeit unter Selbsterwärmung völlig farblos. Nach 60 Min. beträgt die Gewichtszunahme des Kolbens 5 g. Bei einer Gewichtszunahme von insgesamt $10 \mathrm{~g}$ wird die Chlorierung abgebrochen. Man erhält ein farbloses öl, das in Wasser sofort zu Boden sinkt. Das Reaktionsgemisch läßt sich an einer kleinen Kolonne nicht unzersetzt destillieren. Während der Destillation beobachtet man mehr oder minder starke Nebelbildung:
1. Sdp. 75547 bis $95^{\circ}$, farbl. Fl., $7 \mathrm{~g}$

2. „ 95 bis $105^{\circ}$, gelbl. Fl., $10,1 \mathrm{~g}$ (Zersetzung!)

3. „ 80 (Zers.!) bis $138^{\circ}$, gelbl. Fl., $1,8 \mathrm{~g}$

4. Rückstand schwarz, verkohlt.

Frl. Gertrud M e r k el danken wir für die Ausführung der chemischen Kennzahlanalysen. Hr. Prof. $\mathrm{F}$ e h é $\mathrm{r}$ und Frl. $\mathrm{B}$ a u d l e $\mathrm{r}$ hatten die Liebenswürdigkeit, das aus der thermischen Zersetzung des Trimethylenglykol-diacetates erhaltene Allylacetat einer $\mathrm{R}$ a m a n-Analyse zu unterziehen. Auch ihnen sei an - dieser Stelle bestens gedankt.

\title{
Über ein einfaches Verfahren zur Darstellung freier Aminosäureester aus den Esterhydrochloriden
}

\author{
Von Günther Hillmann \\ Aus dem Kaiser-Wilhelm-Institut für Biochemie, Berlin-Dahlem, jetzt Tübingen
}

(Z. Naturforschg. 1, 682-683 [1946]; eingegangen am 25. Juli 1946)

$\mathrm{F}$ ür die Synthese von Peptiden sind meist freie Aminosäureester erforderlich, für deren Darstellung aus den Esterhydrochloriden eine Reihe von Methoden zur Verfügung stehen, die aber z. Tl. umständlich sind oder unbefriedigende Ausbeute liefern. Bei dem bekannten Verfahren von E. Fischer ${ }^{1}$, das auf der Freisetzung von Aminosäureestern durch $\mathrm{NaOH}$ und $\mathrm{K}_{2} \mathrm{CO}_{3}$ beruht, ist die Ausbeute gering (durchschnittlich ca. 50\%). Th. Curtius ${ }^{2}$ stellte die freien Aminosäureester durch Behandlung der Hydrochloride mit $\mathrm{Ag}_{2} \mathrm{O}$ bzw. $\mathrm{AgOH}$ her. Trotz der Modifizierung durch S. Ai und S. Ushida ${ }^{3}$ scheint das Verfahren wegen der Verwendung von $\mathrm{Ag}_{2} \mathrm{O}$ wenig geeignet. Nach E. Abderhalden ${ }^{4}$ werden die Esterhydrochloride mit der genau berechneten Menge Natriumalkoholat umgesetzt; dazu sind relativ große Mengen von absol. Alkohol und Äther notwendig. Beim Abdestillieren des Alkohols werden die Ester der niedrigmolekularen Aminosäuren teilweise mitgerissen. Bei der Einwirkung von $\mathrm{BaO}$ bzw. $\mathrm{Ba}(\mathrm{OH})_{2}$ auf die Esterhydrochloride in Chloroform nach P. A. Levene ${ }^{5}$ wird ein Teil des entstandenen Esters wieder verseift; das gilt vor allem für die besonders alkaliempfindlichen Aminodicarbonsäureester. Nach J. A. B ach$\mathrm{mann}^{6}$ wird diese Fehlerquelle zwar weitgehend durch die Umsetzung mit $\mathrm{BaO}$ in absol. Äther ausgeschaltet, das Verfahren ist jedoch sehr zeitraubend.

Es wurde nun gefunden, daß diese Schwierigkeiten bei der Herstellung freier Aminosäureester sich durch die Umsetzung der Esterhydrochloride mit ammoniakalischem Chloroform vollständig ausschalten lassen. Während die Aminosäureester mit ammoniakalischem

1 Ber. dtsch. chem. Ges. 34, 436 [1901].

2 J. prakt. Chem. [2] 37, 159 [1888].

3 J. Soc. chem. Ind. Japan [Suppl.] 36, 221 [1933].

4 Hoppe-Seylers Z. physiol. Chem. 120, 208 [1922].
Alkohol unter Amidbildung ${ }^{7}$ reagieren, tritt diese Reaktion mit ammoniakalischem Chloroform in der Kälte nicht ein.

Alle Aminosäureester-hydrochloride weisen eine gewisse Löslichkeit in Chloroform auf. Die Umsetzung mit Ammoniak zu freien Aminosäureestern und Ammoniumchlorid findet infolgedessen rasch statt. Da hierbei keine Bildung von Wasser eintritt, besteht keine Gefahr einer Verseifung der freien Ester.

Die Anwendung genau berechneter Mengen von Ammoniak ist nicht erforderlich, da auch bei Vorhandensein eines geringen Utberschusses an Ammoniak unter den ausgearbeiteten Versuchsbedingungen in Chloroform keine Amidbildung stattfindet.

Das bei der Umsetzung entstehende Ammoniumchlorid ist in Chloroform unlöslich und läßt sich bequem abfiltrieren bzw. absaugen. Hierdurch erübrigt sich ein Zusatz von Äther zur besseren Abscheidung, wie es bei der Abderhaldenschen Methode für die Ausfällung des gebildeten Natriumchlorids notwendig ist. Beim Abdestillieren des Chloroforms gehen nur sehr geringe Mengen der niedrig siedenden Aminosäureester mit über. Die Ausbeute ist praktisch quantitativ. Sofern die jeweiligen Versuchsbedingungen bei der Synthese von Peptiden die Verwendung von Lösungen der Aminosäureester in Chloroform zulassen, sind nach dem Abfiltrieren des gebildeten Ammoniumchlorids und der Entfernung des üherschüssigen Ammoniaks im Vakuum die Chloroformlösungen der freien Aminosäureester nach unseren Erfahrungen direkt zur Synthese geeignet.

5 J. Biol. Chem. 81, 699; 86, 419 [1929].

6 Dissertat. Eidgen. T. H. Zürich, Agriculturchem. Labor. 1927.

7 Schenk, Arch. Pharmaz. Ber. dtsch. pharmaz. Ges. 247, 510 [1909]. 


\section{Versuchsteil}

Trockenes Ammoniakgas wird unter Kühlung in trockenes Chloroform bis zur vollständigen Sättigung eingeleitet. Bei $19^{\circ}$ werden in $100 \mathrm{~g}$ Chloroform $2,15 \mathrm{~g}$ Ammoniak gelöst.

Glykokolläthylester. $30 \mathrm{~g}$ Glykokolläthylester-hydrochlorid werden in $25 \mathrm{ccm}$ Chloroform suspendiert und unter Eiskühlung $125 \mathrm{ccm}$ ca. 2-proz. ammoniakalische ChIoroformlösung hinzugegeben. Sodann wird das Reaktionsgemisch etwa 1/4 Stde. abwechselnd gekühlt und geschüttelt. Nach weiterem viertelstündigen Stehenlassen unter Eiskühlung wird das gebildete Ammoniumchlorid abfiltriert bzw. abgesaugt und mit wenig Chloroform gewaschen. Beim Filtrieren empfiehlt sich ein Zusatz von etwas Absorptionskohle zur Chloroformlösung. Das Chloroform wird durch Abdestillieren im Vakuum bei höchstens $20^{\circ}$ Wasserbadtemperatur entfernt und der freie Glykokolläthylester bei $11 \mathrm{~mm} \mathrm{Hg}$ abdestilliert. Ausb. $19 \mathrm{~g}$ (86\% d. Th.).

In analoger Weise wurde der $d, l$-Alaninäthylester in 88-proz. Ausbeute, der d,l-Leucinäthylester bei $0,5 \mathrm{~mm} \mathrm{Hg}$ in 90 -proz. Ausbeute erhalten.

Bei der Herstellung des d,l-Tyrosinäthylesters wurde der nach dem Abdestillieren des Chloroforms sofort kristallisierende Ester nach dem Umkristallisieren aus Benzol in 95-proz. Ausbeute erhalten.

Da das $d, l$-Glutaminsäurediäthylester-hydrochlorid nicht kristallisiert, wurde aus dem gut kristallisierenden $\quad d, l$-Glutaminsäuredimethylester-hydrochlorid $\left(\right.$ Schmp. $=149^{\circ}$ korr. $)$ in der oben genannten Weise der freie $d, l$-Glutaminsäuredimethylester nach dem Abdestillieren des Chloroforms in quantitativer Ausbeute erhalten.

(Bei der Destillation im Hochvakuum tritt immer eine teilweise Zersetzung zu $d, l$-Pyrrolidon-carbonsäuremethylester und Methanol ein.)

\title{
Eine Modifikation der Äthylenbestimmung nach Haber*
}

\author{
Von Hermengild Flaschka \\ Aus dem Kaiser-Wilhelm-Institut für Physikalische Chemie und Elektrochemie, Berlin-Dahlem - \\ (Z. Naturforschg. 1, 683-684 [1946]; eingegangen am 27. Aug. 1946)
}

\begin{abstract}
A. $\mathrm{Noll}^{1}$ hat erstmalig auf die Brauchbarkeit des Chloramin $T^{2}$ in der Maßanalyse hingewiesen und seine Verwendung als Jodersatz vorgeschlagen. Seither wurde eine große Zahl von Titrationsmethoden auf dieser Basis ausgearbeitet. Gestützt auf die vorhandenen Grundlagen wurde eine Modifikation der Äthylenbestimmung nach $\mathrm{H}$ a be $\mathrm{r}^{3}$ entwickelt, bei der Jod, außer in geringsten Mengen, als Indikator nicht verwendet wird.
\end{abstract}

Das Prinzip der Analyse ist folgendes:

In der Bu nteschen Bürette wird aus einer Kaliumbromidbromatlösung durch Säurezusatz Brom freigemacht. Das Brom setzt sich mit dem Athylen um. Der Überschuß an Brom wird durch eine gemessene Menge einer Lösung arseniger Säure reduziert. Die überschüssige arsenige Säure wird mit Chloramin zurücktitriert. An sich wäre es möglich, mit Kaliumbromidbromatlösung zurückzutitrieren ${ }^{4}$. Es ergäbe sich aber daraus der Nachteil, daß gegen Ende der Titration sehr lang-

\footnotetext{
* Eingereicht bei der Zeitschrift für analyt. Chemie im Dezember 1944, dort jedoch nicht mehr erschienen.

1 Papierfabrikant 22, 385.

2 -Toluolsulfochloramid-natrium.
}

sam $\mathrm{zu}$ titrieren ist (Methylorange als Indikator wird durch freiwerdendes Brom zerstört; Zeitreaktion!) oder daß die Lösung auf 70 bis $80^{\circ} \mathrm{zu}$ erwärmen wäre ${ }^{5}$, was besonders bei Serienanalysen einen Zeitverlust mit sich bringt.

\section{Durchführung der Analyse}

50 bis $70 \mathrm{ccm}$ Analysengas werden in die B unte sche Bürette gesaugt. Bei geschlossenem oberen Hahn wird das noch vorhandene Sperrwasser bis zur Marke -10 abgesaugt, wozu man sich am besten einer Wasserstrahlpumpe bedient. Aus einem flachen Schälchen läßt man nun durch den unteren Hahn $20 \mathrm{ccm}$ 0,1-n. Kaliumbromidbromat einströmen. Sodann saugt man noch $5 \mathrm{ccm}$ Salzsäure (1:1) nach und schüttelt bis zur völligen Absorption des Äthylens, die nach ungefähr 8 Min. erreicht ist.

Nun füllt man mit einer Pipette $25 \mathrm{ccm} 0,1-n$. arsenige Säure in den Trichter der Bunteschen Bürette und läßt sie durch Öffnen des oberen Hahnes in das Innere strömen. Wenn nach kurzem Schütteln die Flüssigkeit farblos geworden ist, läßt man sie nach unten in einen Kolben ab und spült mit Wasser nach.

Die Lösung versetzt man mit 2 bis $3 \mathrm{~g}$ Natrium-

3 Tre a d we ll, Kurzes Lehrbuch der analyt. Chemie II, 11. Aufl. 1941, S. 721.

${ }^{4}$ St. G y ö r g y, Z. analyt. Chem. 1893, 415.

$5 \mathrm{~W}$. B r a chmann, Dissertat. Breslau 1927. 